\title{
EDITORIAL
}

\section{THE MULTIDIMENSIONALITY OF FRAILTY: MANY FACES OF ONE SINGLE DICE}

\author{
M. CESARI
}

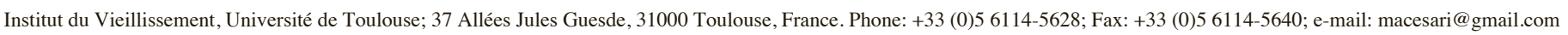

The present special issue of the Journal of Nutrition Health and Aging is focused on the theme of "frailty and cognitive decline". Among its contributions (including methodological, epidemiological, and intervention studies), it is well-evident how difficult it is doing research (and, consequently, clinics) in older persons. What it is clear, direct, and straigthforward in the medicine targeting youngs and adults, becomes unfocused, complex, and contraddictory in elders. After all, this issue is well-known and at the basis of the difficulties in implementing "evidence-based guidelines" to older persons. It is not aim of the present editorial paper to discuss the nature of this problem or its consequences, since a large body of literature is already available on the topic $(1,2)$. It is sufficient to accept that geriatric research and medicine move themselves within a completely new field requiring different roles compared to other disciplines. To understand why a certain condition is related to a well-identified outcome in the adulthood, researchers and clinicians usually focus their efforts targeting one or few specific underlying pathophysiological pathways. In geriatrics, this is more complex because the studied conditions are frequently resulting from a heterogeneous combination of multiple actors, including socio-demographic, behavioral, biological, and enviromental factors. Furthermore, the first issue challenging the work of researchers and clinicians in this field is to demarcate the subtle difference between "aging" and "disease", an unclear threshold largely determined by diagnostic accuracy and arbitrary decisions.

Differently from other specialties, geriatric medicine can also be considered as relatively recent. For instance, it is noteworthy that researchers have started at systematically exploring the frailty syndrome (a major condition characterizing advanced age) only about 20 years ago, or that the term "sarcopenia" (another relevant matter for geriatrics) was originally coined only in the late Eighties. Consequently, we are still at a very early phase of the comprehension of the aging process and its related conditions, and struggle to delineate at our best those conditions that currently appear more promising in counteracting the disabling process.

Frailty is one of the most relevant geriatric conditions which has shown to be extremely suitable to serve as target for preventive and therapeutical interventions. Nevertheless, to date, what we all agree is only that it is a detrimental state exposing the older person to adverse events due to an increased vulnerability to endogenous and exogenous stressors (3). Then, when this theorical foundation is tried to be translated into practice, it generates a huge controversy about the most appropriate operative definition to apply. As clearly explained by Panza and colleagues in their review paper (4), the operative conceptualization of frailty cannot ignore its multiple constituing domains leading towards the need of a holistic definition of it. Unfortunately, we do not know yet which of the many already existing operative definitions is more suitable to objectively summarize this complex condition into a "number". Independently of which it is (or will be), we need to admit that every "clinical-friendly definition" to be adopted will likely never be totally exhaustive.

Interestingly but perhaps not totally surprisingly, the paper by Drame and colleagues (5) reports an extremely poor agreement among four different frailty assessment instruments. Does this mean that none of the instruments is valid at capturing frailty? Surely not. It is more likely that each of them is measuring a partial and/or specific aspect of this multifaceted syndrome, as suggested by the subsequent significant and consistent results obtained by all the tools in predicting negative events (i.e., one-year institutionalization and mortality). Consistently, if the article by Jacobs and colleagues (6) shows that even a fifth definition of frailty (the most commonly used proposed by Fried and colleagues (7)) is predictive of mortality, previous studies already reported only a moderate correlation of this same index with other frailty assessment tools (8).

The multidimensionality of the frailty syndrome can be easily appreciated in the papers by Auyeung and colleagues (9) and Avila-Funes and colleagues (10) included in the present issue of the Journal of Nutrition Health and Aging. In fact, from different perspectives and using different analytical approaches, both studies tend to support the idea that cognition and physical function are independent domains equally contributing to the same common pathway of the disabling process (11). This may mean that at least these two domains should be part of the frailty assessment. But then, what about nutrition, physical activity, depressive symptoms, clinical conditions, socioeconomic status...?

The existence of multiple operative definitions poses the real risk of losing specificity towards the condition of interest. It may simply be that the "frailty status" differently identified by each single definition is consistently associated with negative health-related events simply because what is "bad" is constantly compared to what is "good". In other words, it might not be frailty per se at inducing the profile of increased risk, but the collection of multiple and independent risk factors. In this context, as mentioned above, it cannot be ignored that frailty is 


\section{THE MULTIDIMENSIONALITY OF FRAILTY: MANY FACES OF ONE SINGLE DICE}

today commonly considered as a syndrome. As such, all the manifestations associated with it occur in combination, and no single manifestation is sufficient to identify those within the syndrome. At the same time, the multidimensional nature of the syndrome (which is resembling at a pre-clinical level the complexity of the geriatric patient) indeed requires a comprehensive evaluation to be measured, and multidimensional interventions to prevent or treat it.

This need of simultaneously acting at different levels and on multiple targets is implied in the rationale leading Kamphuis and colleagues (12) at evaluating the effects of a multinutrient drink (including omega-3 fatty acids, antioxidants, B vitamins, and choline) on cognition. Again, should we pursue the traditional methodology targeting one specific aspect at the time even when evaluating complex geriatric conditions (with the risk of obtaining false negative results simply because the possible benefits are overwhelmed by the "catastrofic" scenario of the geriatric patient), or more pragmatically test combinations of interventions (with the consequent difficulties in appreciating the specific effects of each tested component on the final outcome)? Hopefully, such doubts will be cleared in future when individual-tailored interventions will be developed, for example, on the basis of systems biology (13). In the meanwhile, a possible intermediate solution might be that clinical trials focused on geriatric conditions may start targeting multiple aspects of the disease (i.e., through multidomain interventions) while being specifically guided towards the single participant's needs (e.g., according to a preliminary comprehensive geriatric assessment).

Parallely to the frailty syndrome, another "hot topic" for geriatric research and medicine is sarcopenia (14). Sarcopenia (in its wider significance) is commonly considered as a hallmark of aging and a typical feature of the frailty syndrome. These two conditions are not only closely related each other, but also share similar methodological issues $(15,16)$. In fact, whereas both characterize the aging process and have multifactorial etiology, they also currently lack of unique standardized and objective definitions. For example, while Bautmans and colleagues (17) are more interested at evaluating qualitative measures of sarcopenia, Wirth and colleagues are specifically focused on quantitative parameters of body composition (18). In a third paper included in this issue, Canon and Crimmins (19) define sarcopenia (or "muscle quality") on the basis of a ratio between muscle strength and mass (thus, simultaneously capturing both the dimensions of the sarcopenia phenomenon). Although completely different among them, all these approaches may yet be considered legitimate to explore the common theme of sarcopenia. In fact, they still provide useful and needed insights on different operative and theoretical aspects of sarcopenia which are crucial in this preliminary phase of research. The study of this condition (or "syndrome" for many (20)) is too promising to be slowed-down by methodological doubts. The adoption of scientifically-sound devices aimed at capturing the inner nature of the studied syndrome and, at the same time, assessing a range of its constituing aspects may well and adequately support the propedeutical scientific acquisitions towards future more accurate definitions. When Phase III trials on sarcopenia (or frailty, too) will start being developed in the next future, more rigorous criteria (released as consensual statements by international working groups/task forces and based on the evidence accumulated over these years) will then be required.

In conclusion, we all well-know that a "clear" clinical condition as described in the medicine textbooks will be hardly found in the geriatric patient, because there will be always another (or, more easily, several others) factor(s) influencing the health status and the final outcomes. After all, this is what distinguishes an "old" from a "geriatric" patient. The contraddictions and uncertainties raised by the initial explorations of the major geriatric syndromes (such as frailty and sarcopenia) performed in these last years will provide the basis on which building up consensual statements and guidelines in the very next future.

\section{References}

1. Tonelli MR. The philosophical limits of evidence-based medicine. Acad Med. 1998; 73:1234-1240.

2. Scott IA, Guyatt GH. Cautionary tales in the interpretation of clinical studies involving older persons. Arch Intern Med. 2010;170:587-595.

3. Abellan van Kan G, Rolland Y, Bergman H, Morley JE, Kritchevsky SB, Vellas B. The I.A.N.A Task Force on frailty assessment of older people in clinical practice. J Nutr Health Aging. 2008;12:29-37.

4. Panza F, Solfrizzi V, Frisardi V, Maggi S, Sancarlo D, Addante F et al. Different models of frailty in predementia and dementia syndromes. J Nutr Health Aging 2011;15(8):711-719.

5. Drame M, Novella JM, Jolly D, Laniece I, Somme D, Heitz D et al. Rapid cognitive decline, one-year institutional admission and one-year mortality: analysis of the ability to predict and inter-tool agreement of four validated clinical frailty indexes in the SAFEs cohort. J Nutr Health Aging 2011;15(8):699-705.

6. Jacobs JM, Cohen A, Ein-Mor E, Maaravi Y, Stessman J. Frailty, cognitive impairment and mortality among the oldest old. J Nutr Health Aging 2011;15(8):678-682.

7. Fried LP, Tangen CM, Walston J, Newman AB, Hirsch C, Gottdiener J et al. Frailty in older adults: evidence for a phenotype. J Gerontol A Biol Sci Med Sci. 2001;56:M146-56.

8. Rockwood K, Andrew M, Mitnitski A. A comparison of two approaches to measuring frailty in elderly people. J Gerontol A Biol Sci Med Sci. 2007;62:738-743.

9. Auyeung TW, Lee JSW, Kwok T, Woo J. Physical frailty predicts cognitive decline - A four-year prospective study in 2737 cognitively normal older adults. J Nutr Health Aging 2011;15(8):690-694.

10. Avila-Funes JA, Pina-Escudero SD, Aguilar-Navarro S, Gutierrez-Robledo LM, RuizArregui L, Amieva H. Cognitive impairment and low physical activity are the components of frailty more strongly associated with disability. J Nutr Health Aging 2011;15(8):683-689.

11. Atkinson HH, Rosano C, Simonsick EM, Williamson JD, Davis C, Ambrosius WT et al. Cognitive function, gait speed decline, and comorbidities: the health, aging and body composition study. J Gerontol A Biol Sci Med Sci. 2007;62:844-850.

12. Kamphuis PJGH, Verhey FRJ, Olde Rikkert MGM, Twisk JWR, Swinkels SHN, Scheltens P. Efficacy of a medical food cognition in Alzheimer's disease: results from secondary analyses of a randomized, controlled trial. J Nutr Health Aging 2011;15(8):720-724.

13. Nicholson JK, Wilson ID. Opinion: understanding 'global' systems biology: metabonomics and the continuum of metabolism. Nat Rev Drug Discov. 2003;2:668-676.

14. Chumlea WC, Cesari M, Evans WJ, Ferrucci L, Fielding RA, Pahor M et al. Sarcopenia: designing phase IIb trials: international working group on sarcopenia. J Nutr Health Aging. 2011;15:450-455.

15. Pahor M, Cesari M. Designing Phase II B trials in sarcopenia: the best target population. $\mathrm{J}$ Nutr Health Aging 2011;15(8):725-730.

16. Conroy S. Editorial: Defining Frailty - The Holy Grail of Geriatric Medicine. J Nutr Health Aging. 2009;13:389.

17. Bautmans I, Onyema O, Van Puyvelde K, Pleck S, Mets T. Grip work estimation during sustained maximal contraction: validity and relationship with dependency and inflammation in elderly persons. J Nutr Health Aging 2011;15(8):731-736.

18. Wirth R, Smoliner C, Sieber CC, Volkert D. Cognitive function is associated with body composition and nutritional risk of geriatric patients. J Nutr Health Aging 2011;15(8):706-710.

19. Canon ME, Crimmins EM. Sex differences in the association between muscle quality, inflammatory markers, and cognitive decline. J Nutr Health Aging 2011;15(8):695-698.

20. Rosenberg IH. Sarcopenia: origins and clinical relevance. J Nutr. 1997;127:990S-991S 